\title{
Wormholes in a Viable $f(T)$ Gravity
}

\author{
Mubasher Jamil, ${ }^{1,2}$ Davood Momeni, ${ }^{2}$ and Ratbay Myrzakulov ${ }^{2}$ \\ ${ }^{1}$ Center for Advanced Mathematics and Physics (CAMP), \\ National University of Sciences and Technology (NUST), H-12, Islamabad, Pakistan \\ ${ }^{2}$ Eurasian International Center for Theoretical Physics, \\ Eurasian National University, Astana 010008, Kazakhstan
}

\begin{abstract}
In this paper, we derive some new exact solutions of static wormholes in $f(T)$ gravity. We discuss independent cases of the pressure components including isotropic and anisotropic pressure. Lastly we consider radial pressure satisfying a barotropic equation of state. We also check the behavior of null energy condition (NEC) for each case and observe that it is violated for anisotropic while it is satisfied for isotropic and barotropic cases.
\end{abstract}

Keywords: Wormhole; Torsion; Modified Gravity; Energy conditions.

PACS numbers: 04.50.-h, 04.50.Kd, 04.20.Jb 


\section{INTRODUCTION}

Teleparallelism was proposed by Einstein as an attempt to unify electromagnetism and gravity. But as time rolled on scientists lost interest in this concept of unification and as a result, today teleparallelism is just considered as an alternative classical gauge theory for gravity. It corresponds to a diffeomorphism invariant gauge model for the boost Poincare's group [1]. The crucial new idea, for Einstein, was the introduction of an orthogonal basis, called the tetrads. Here the space-time is characterized by a curvature-free linear connection, called the Weitzenbock connection defining the scalar torsion without Riemannian curvature. In general relativity (GR), we use curvature to unify the geometry and the gravitational field according to the Mach's equivalence principle, thus successfully describing the gravitational interaction. Teleparallelism, explains gravitation by torsion. Therefore it seems to be just a matter of convention that whether gravity needs a curved spacetime. Exact solutions for this newly proposed theory play a key role to test the validity of the results as well as an attempt to find the correct path of the test particles in the curved spacetime, based on the torsion. We are focused in this paper on wormhole solutions, as a class of exact solutions with a little different aspect. A typical spherically symmetric spacetime can be described as a wormhole and as a bridge between two distinct parts of the spacetime (see [2] for a review). It was first proposed by Morris and Thorne [3] in the context of the time machine idea. In literature, wormholes have been discussed [4], [5], [6]. Otherwise, to explain the new challenge of the cosmology, the modified gravity has gained enormous popularity in the cosmological society since it passes several solar system and astrophysical tests successfully [8]. In addition to this, modified gravity models can be used as well, for the recent cosmic acceleration without resorting to any form of DE. For example some popular models like Loop quantum gravity [9], extra dimensional braneworlds [10], $f(R)$ [11], $f(T)[12$ 15].

In this paper, we derive some new exact solutions of static wormholes in $f(T)$ gravity. We discuss independent cases of the pressure components including isotropic and anisotropic pressure. Lastly we consider the non zero radial pressure $p_{r}(r)$ satisfying a typical barotropic equation of state. We also check the behavior of null energy condition (NEC) for each case and observe that it is violated for anisotropic while it is satisfied for isotropic and barotropic cases.

The plan of this paper is as follows. In section II, the basic equations for the $f(T)$ model is discussed. In section III, the field equations are written. In section IV, wormhole solutions in $f(T)$ gravity are discussed. In section $\mathrm{V}$, we propose our viable $f(T)$ model. In section VI, an exact anisotropic solution is derived. In section VII, the isotropic solutions are derived. In section 
VIII, the barotropic solutions are investigated. Finally we end with some concluding remarks in section IX.

\section{GRAVITATIONAL FIELD EQUATIONS IN A F(T) GRAVITY}

We assume that the metric is static spherically symmetric in the usual Schwarzschild coordinates in the following form [22],

$$
d s^{2}=e^{a(r)} d t^{2}-e^{b(r)} d r^{2}-r^{2}\left(d \theta^{2}+\sin ^{2} \theta d \varphi^{2}\right),
$$

Here $a(r)$ and $b(r)$ are the unknown components of the diagonal metric. We take the orthogonal but off-diagonal tetrads to avoid the teleparallel gravity (TEGR) with $f_{T T}=0$, given by [22]

$$
e_{\mu}^{i}=\left(\begin{array}{cccc}
e^{a / 2} & 0 & 0 & 0 \\
0 & e^{b / 2} \sin \theta \cos \phi & r \cos \theta \cos \phi & -r \sin \theta \sin \phi \\
0 & e^{b / 2} \sin \theta \sin \phi & r \cos \theta \sin \phi & r \sin \theta \cos \phi \\
0 & e^{b / 2} \cos \theta & -r \sin \theta & 0
\end{array}\right) .
$$

Also $\operatorname{det}\left(e^{i}{ }_{\mu}\right)$ reads $e=\exp ((a+b) / 2) r^{2} \sin \theta$. Further the scalar torsion $T$ is

$$
T(r)=\frac{2 e^{-b}\left(e^{b / 2}-1\right)\left(e^{b / 2}-1-r a^{\prime}\right)}{r^{2}}
$$

respectively.

The equations of motion are the following [22]

$$
\begin{aligned}
4 \pi \rho(r) & =\frac{e^{-b / 2}}{r}\left(1-e^{-b / 2}\right) T^{\prime} f_{T T}-\left(\frac{T}{4}-\frac{1}{2 r^{2}}\right) f_{T}+\frac{e^{-b}}{2 r^{2}}\left(r b^{\prime}-1\right) f_{T}-\frac{f}{4}, \\
4 \pi p_{r}(r) & =\left[-\frac{1}{2 r^{2}}+\frac{T}{4}+\frac{e^{-b}}{2 r^{2}}\left(1+r a^{\prime}\right)\right] f_{T}-\frac{f}{4}, \\
4 \pi p_{t}(r) & =\frac{e^{-b}}{2}\left(\frac{a^{\prime}}{2}+\frac{1}{r}-\frac{e^{b / 2}}{r}\right) T^{\prime} f_{T T}+f_{T}\left\{\frac{T}{4}+\frac{e^{-b}}{2 r}\left[\left(\frac{1}{2}+\frac{r a^{\prime}}{4}\right)\left(a^{\prime}-b^{\prime}\right)+\frac{r a^{\prime \prime}}{2}\right]\right\}-\frac{f}{4},
\end{aligned}
$$

where $\rho(r), p_{r}(r), p_{t}(r)$ are the energy density, the radial pressure and the pressure. The above system of field equations (3)-(5) is not closed. Consequently we fix some of these functions.

\section{WORMHOLE SOLUTIONS IN $f(T)$ GRAVITY}

For a better understanding of numerous features of the wormhole geometry, we shall use the following redefinition of the metric function

$$
e^{-b(r)}=1-\frac{\beta(r)}{r} .
$$


In the theory of wormholes, $a(r)$ and $\beta(r)$ are arbitrary functions of the radial coordinate. In brief in the $3+1$ decomposition language, $a(r)$ denotes the redshift function, which is related to the gravitational redshift $g_{00}$. Also $\beta(r)$ defines the shape function and it specifies the geometrical shape of the stationary wormhole [24]. The coordinate $r$ is in the strip $r_{0}<r<+\infty, r_{0}$ denotes the radius of wormhole's throat. By flaring out of the throat, we mean to impose the condition $\left(\beta-\beta^{\prime} r\right) / 2 \beta^{2}>0[24]$ and at the throat location we observe that $\beta^{\prime}\left(r_{0}\right)<1$.

It turns out that that Morris-Thorne wormholes disobey all energy conditions and averaged null energy condition. In fact, the weak energy condition (WEC) proposes that the energy density must remain locally positive definite, i.e.

$$
\rho=T_{\mu \nu} U^{\mu} U^{\nu} \geq 0
$$

for all timelike vector fields $U^{\mu}$, where $T_{\mu \nu}$ is the usual energy-momentum (EM) tensor. In the limit of the quantum effects, this expression of the EM tensor can be replaced by the semi classical quantum expectation value $\left\langle T_{\mu \nu}^{v a c c u a}>\right.$, where it can be replaced as a source term in the right hand side of the gravitational field equation. For example, this kind of EM tensors with conformal anomaly can produce a rich family of the exact $(\mathrm{A}) \mathrm{dS}$ black holes in the context of the $f(R)$ gravity [25].

Further we can show that by replacing the general expression of the EM tensor for a typical Lorentz invariance Lagrangian $\mathcal{L}$, by the expression

$$
T_{\mu \nu}=g_{\mu \nu} \mathcal{L}-2 \frac{\delta \mathcal{L}}{\delta g^{\mu \nu}}
$$

and by adopting a timelike velocity vector stream by $U^{\mu}=\phi^{; \mu}$, again this new expression remains positive definite. In the locally Lorentzian frame of the matter, for example in the co moving frame in the perfect fluids, this means $\rho>0$ and $\rho+p_{i} \geq 0$, where $i=r, t$. The WEC implies NEC, i.e. the null energy condition: $T_{\mu \nu} k^{\mu} k^{\nu} \geq 0$, here $k^{\mu}$ denotes a null vector field [26]. Our goal is to find some exact solutions for wormholes and check whether they satisfy or violate the above energy conditions. We briefly review the usual energy conditions in the next section.

\section{ENERGY CONDITIONS}

The energy conditions are used in different contexts to derive variety of general results which hold for different physical situations. Under these conditions, one allows not just gravity to be attractive but also the energy density to be positive and flows not to be faster than light [20]. The 
notion of energy conditions arise from the Raychaudhuri equation, given by

$$
\frac{d \theta}{d \tau}=-\frac{1}{3} \theta^{2}-\sigma_{\mu \nu} \sigma^{\mu \nu}+\omega_{\mu \nu} \omega^{\mu \nu}-R_{\mu \nu} u^{\mu} u^{\nu}
$$

where $u^{\mu}$ is a vector field representing the congruence of timelike geodesics. Also $R_{\mu \nu}$, represent Ricci tensor $\theta$,the expansion parameter, $\sigma_{\mu \nu}$ the shear and $\omega_{\mu \nu}$, the rotation associated with the congruence respectively. For a congruence of null geodesics, $k^{\mu}$, we get

$$
\frac{d \theta}{d \tau}=-\frac{1}{2} \theta^{2}-\sigma_{\mu \nu} \sigma^{\mu \nu}+\omega_{\mu \nu} \omega^{\mu \nu}-R_{\mu \nu} k^{\mu} k^{\nu}
$$

which is called the Raychaudhuri equation. The essence of such checking energy conditions is independent of any gravity theory and that these are purely geometrical (for a review on the energy conditions, see the classic text [21]).

The null energy condition (NEC), weak energy condition (WEC), strong energy condition (SEC) and the dominant energy condition (DEC) are respectively given by [22, 23]

$$
\begin{aligned}
\mathrm{NEC} & \Longleftrightarrow \rho_{\mathrm{eff}}+p_{\mathrm{eff}} \geq 0 . \\
\mathrm{WEC} & \Longleftrightarrow \rho_{\mathrm{eff}} \geq 0 \text { and } \rho_{\mathrm{eff}}+p_{\mathrm{eff}} \geq 0 . \\
\mathrm{SEC} & \Longleftrightarrow \rho_{\mathrm{eff}}+3 p_{\mathrm{eff}} \geq 0 \text { and } \rho_{\mathrm{eff}}+p_{\mathrm{eff}} \geq 0 . \\
\mathrm{DEC} & \Longleftrightarrow \rho_{\mathrm{eff}} \geq 0 \text { and } \rho_{\mathrm{eff}} \pm p_{\mathrm{eff}} \geq 0,
\end{aligned}
$$

where $\rho_{\text {eff }}$ and $p_{\text {eff }}$ are respectively the effective energy density and pressure. The matter supporting the wormhole geometry is termed "exotic" matter if it violates the above energy conditions, particularly the NEC, as its violation leads to the violation of all the remaining energy conditions $[?]$.

\section{EQUATIONS OF MOTION FOR A VIABLE MODEL OF $f(T)$}

We must fix the form of the $f(T)$ to simplify the equations of motion and to find a class of the solutions, corresponds to the wormhole. Recently we proposed a physically reasonable expression for $f(T)[27]$ in the following form,

$$
f(T)=2 c_{1} \sqrt{-T}+\alpha T+c_{2}
$$

Here $\left\{\alpha, c_{1}, c_{2}\right\}$ are arbitrary constants but the values must be obtain by some additional cosmological evidences.

Now field equations (3)-(15) and (13) give us the following system of equation of motion 


$$
\begin{aligned}
& 4 \pi \rho(r)=\frac{1}{8} \mathrm{e}^{-b}\left(-1+\mathrm{e}^{-\frac{1}{2} b}\right) \sqrt{2} \mathrm{e}^{-\frac{1}{2} b}\left(-4 \mathrm{e}^{b}+\left(-2 r^{2} a^{\prime \prime}+\left(2 r+b^{\prime} r^{2}\right) a^{\prime}+8+2 r b^{\prime}\right) \mathrm{e}^{\frac{1}{2} b}+2 r^{2} a^{\prime \prime}\right. \\
& \left.+\left(-2 b^{\prime} r^{2}-2 r\right) a^{\prime}-2 r b^{\prime}-4\right) c_{1}\left(\frac{\mathrm{e}^{-b}\left(\mathrm{e}^{\frac{1}{2} b}-1\right)\left(\mathrm{e}^{\frac{1}{2} b}-1-r a^{\prime}\right)}{r^{2}}\right)^{-\frac{3}{2}} r^{-3} \\
& -\left(\frac{1}{2} c_{1} \sqrt{2} \frac{1}{\sqrt{\frac{\mathrm{e}^{-b}\left(\mathrm{e}^{\frac{1}{2} b}-1\right)\left(\mathrm{e}^{\frac{1}{2} b}-1-r a^{\prime}\right)}{r^{2}}}}+\alpha\right)\left(\frac{1}{2} \frac{\mathrm{e}^{-b}\left(\mathrm{e}^{\frac{1}{2} b}-1\right)\left(\mathrm{e}^{\frac{1}{2} b}-1-r a^{\prime}\right)}{r^{2}}-\frac{1}{2} r^{-2}\right) \\
& -\frac{1}{2} c_{1} \sqrt{2} \sqrt{\frac{\mathrm{e}^{-b}\left(\mathrm{e}^{\frac{1}{2} b}-1\right)\left(\mathrm{e}^{\frac{1}{2} b}-1-r a^{\prime}\right)}{r^{2}}}-\frac{1}{2} \frac{\alpha \mathrm{e}^{-b}\left(\mathrm{e}^{\frac{1}{2} b}-1\right)\left(\mathrm{e}^{\frac{1}{2} b}-1-r a^{\prime}\right)}{r^{2}} \\
& -\frac{1}{4} c_{2}+\frac{1}{4} \mathrm{e}^{-b}\left(c_{1} \sqrt{2} \frac{1}{\sqrt{\frac{\mathrm{e}^{-b}\left(\mathrm{e}^{\frac{1}{2} b}-1\right)\left(\mathrm{e}^{\frac{1}{2} b}-1-r a^{\prime}\right)}{r^{2}}}}+2 \alpha\right)\left(r b^{\prime}-1\right) r^{-2}
\end{aligned}
$$

$$
\begin{aligned}
& 4 \pi p_{r}(r)=-\frac{1}{4}\left(\left(\left(-2 \alpha-2 \alpha r a^{\prime}\right) \mathrm{e}^{-b}+r^{2} c_{2}+2 \alpha\right) \sqrt{\frac{\mathrm{e}^{-b}\left(\mathrm{e}^{\frac{1}{2} b}-1\right)\left(\mathrm{e}^{\frac{1}{2} b}-1-r a^{\prime}\right)}{r^{2}}}\right. \\
& \left.-\left(-1-\left(\mathrm{e}^{\frac{1}{2} b}\right)^{2} \mathrm{e}^{-b}+\mathrm{e}^{-b}\left(2+r a^{\prime}\right) \mathrm{e}^{\frac{1}{2} b}\right) \sqrt{2} c_{1}\right) \frac{1}{\sqrt{\frac{\mathrm{e}^{-b}\left(\mathrm{e}^{\frac{1}{2} b}-1\right)\left(\mathrm{e}^{\frac{1}{2} b}-1-r a^{\prime}\right)}{r^{2}}}} r^{-2},
\end{aligned}
$$

$$
\begin{aligned}
4 \pi p_{t}(r)= & \frac{1}{8} \frac{1}{\sqrt{\frac{\mathrm{e}^{-b\left(\mathrm{e}^{\frac{1}{2} b}-1\right)\left(\mathrm{e}^{\frac{1}{2} b}-1-r a^{\prime}\right)}}{r^{2}}} r^{-2} \times\left(r\left(\alpha\left(2 r a^{\prime \prime}+\left(a^{\prime}-b^{\prime}\right)\left(2+r a^{\prime}\right)\right) \mathrm{e}^{-b}-2 r c_{2}\right)\right.} \\
& \times \sqrt{\frac{\mathrm{e}^{-b\left(\mathrm{e}^{1 / 2 b}-1\right)\left(\mathrm{e}^{\frac{1}{2} b}-1-r a^{\prime}\right)}}{r^{2}}}+\frac{1}{2}\left(-4 \mathrm{e}^{b}+\left(4 r a^{\prime}+8\right) \mathrm{e}^{\frac{1}{2} b}+2 r^{2} a^{\prime \prime}\right. \\
& \left.\left.+r^{2} a^{\prime 2}+\left(-2 r-b^{\prime} r^{2}\right) a^{\prime}-2 r b^{\prime}-4\right) \mathrm{e}^{-b} \sqrt{2} c_{1}\right) .
\end{aligned}
$$

Now we will discuss the possible physical solution in the following three cases.

1. Anisotropic fluid: $p_{r}(r) \neq p_{t}(r)$.

2. Isotropic fluid: $p_{r}(r)=p_{t}(r)$.

3. Barotropic EoS: $p_{r}(r)=k \rho(r)$. 


\section{ANISOTROPIC SOLUTION}

In the first class for exact solution with anisotropic components in the EM tensor of the matter fields, We assume that [22]

$$
a(r)=c_{1}, \quad b(r)=-\log \left(1-\left(\frac{r_{0}}{r}\right)^{n+1}\right),
$$

where $c_{1}, n$ and $r_{0}$ are positive constants. Inserting these functions (17) into the EM tensor system, Eqs. (3)-(15), provides the following solutions

$$
\begin{aligned}
& 4 \pi \rho(r)=\frac{1}{4}\left(c_{1} \sqrt{2}\left(\frac{r_{0}}{r}\right)^{n+1} n r+3 c_{1} \sqrt{2}\left(\frac{r_{0}}{r}\right)^{n+1}-2 \alpha \sqrt{\left(-1+\sqrt{1-\left(\frac{r_{0}}{r}\right)^{n+1}}\right)^{2} r^{-2}}\left(\frac{r_{0}}{r}\right)^{n+1} n\right. \\
& +4 \alpha \sqrt{\left(-1+\sqrt{1-\left(\frac{r_{0}}{r}\right)^{n+1}}\right)^{2} r^{-2}}\left(\frac{r_{0}}{r}\right)^{n+1}+3 c_{1} \sqrt{2} r\left(\frac{r_{0}}{r}\right)^{n+1}-c_{1} \sqrt{2}\left(\frac{r_{0}}{r}\right)^{n+1} n
\end{aligned}
$$

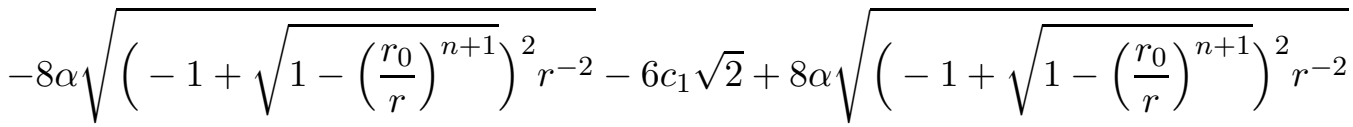

$$
\begin{aligned}
& \times \sqrt{1-\left(\frac{r_{0}}{r}\right)^{n+1}}-2 c_{1} \sqrt{2} r+6 \sqrt{1-\left(\frac{r_{0}}{r}\right)^{n+1}} c_{1} \sqrt{2}-c_{2} r^{2} \sqrt{\left(-1+\sqrt{1-\left(\frac{r_{0}}{r}\right)^{n+1}}\right)^{2} r^{-2}} \\
& \left.+2 \sqrt{1-\left(\frac{r_{0}}{r}\right)^{n+1}} c_{1} \sqrt{2} r\right) r^{-2} \frac{1}{\sqrt{\left(-1+\sqrt{1-\left(\frac{r_{0}}{r}\right)^{n+1}}\right)^{2} r^{-2}}}, \\
& 4 \pi p_{r}(r)=-\frac{1}{4}\left(2 \alpha \sqrt{\left(-1+\sqrt{1-\left(\frac{r_{0}}{r}\right)^{n+1}}\right)^{2} r^{-2}}\left(\frac{r_{0}}{r}\right)^{n+1} \sqrt{1-\left(\frac{r_{0}}{r}\right)^{n+1}}\right. \\
& +\sqrt{\left(-1+\sqrt{1-\left(\frac{r_{0}}{r}\right)^{n+1}}\right)^{2} r^{-2}} \sqrt{1-\left(\frac{r_{0}}{r}\right)^{n+1}} c_{2} r^{2}+2 \sqrt{1-\left(\frac{r_{0}}{r}\right)^{n+1}} c_{1} \sqrt{2}-2 c_{1} \sqrt{2} \\
& \left.+2 c_{1} \sqrt{2}\left(\frac{r_{0}}{r}\right)^{n+1}\right) \frac{1}{\sqrt{\left(-1+\sqrt{1-\left(\frac{r_{0}}{r}\right)^{n+1}}\right)^{2} r^{-2}}} \frac{1}{\sqrt{1-\left(\frac{r_{0}}{r}\right)^{n+1}}} r^{-2},
\end{aligned}
$$




$$
\begin{aligned}
4 \pi p_{t}(r)= & \frac{1}{8}\left(2 \alpha \sqrt{\left(-1+\sqrt{1-\left(\frac{r_{0}}{r}\right)^{n+1}}\right)^{2} r^{-2}}\left(\frac{r_{0}}{r}\right)^{n+1} \sqrt{1-\left(\frac{r_{0}}{r}\right)^{n+1}} n\right. \\
& +2 \alpha \sqrt{\left(-1+\sqrt{1-\left(\frac{r_{0}}{r}\right)^{n+1}}\right)^{2} r^{-2}}\left(\frac{r_{0}}{r}\right)^{n+1} \sqrt{1-\left(\frac{r_{0}}{r}\right)^{n+1}} \\
& -2 \sqrt{\left(-1+\sqrt{1-\left(\frac{r_{0}}{r}\right)^{n+1}}\right)^{2} r^{-2}} \sqrt{1-\left(\frac{r_{0}}{r}\right)^{n+1}} c_{2} r^{2}-4 \sqrt{1-\left(\frac{r_{0}}{r}\right)^{n+1}} c_{1} \sqrt{2}+4 c_{1} \sqrt{2} \\
& \left.-4 c_{1} \sqrt{2}\left(\frac{r_{0}}{r}\right)^{n+1}+c_{1} \sqrt{2}\left(\frac{r_{0}}{r}\right)^{n+1} n \sqrt{1-\left(\frac{r_{0}}{r}\right)^{n+1}}+3 \sqrt{1-\left(\frac{r_{0}}{r}\right)^{n+1}} c_{1} \sqrt{2}\left(\frac{r_{0}}{r}\right)^{n+1}\right) \\
& \times \frac{1}{\sqrt{\left(-1+\sqrt{1-\left(\frac{r_{0}}{r}\right)^{n+1}}\right)^{2} r^{-2}}} \frac{\sqrt{1-\left(\frac{r_{0}}{r}\right)^{n+1}}}{r^{-2}} \cdot
\end{aligned}
$$

The energy conditions read

$$
\begin{aligned}
& \rho+p_{r}=\frac{1}{16 \pi} \frac{1}{\sqrt{\left(-1+\sqrt{1-\left(\frac{r_{0}}{r}\right)^{n+1}}\right)^{2} r^{-2}}} \frac{1}{\sqrt{1-\left(\frac{r_{0}}{r}\right)^{n+1}}} r^{-2}\left(\sqrt{1-\left(\frac{r_{0}}{r}\right)^{n+1}} c_{1} \sqrt{2}\left(\frac{r_{0}}{r}\right)^{n+1} n r\right. \\
& +3 \sqrt{1-\left(\frac{r_{0}}{r}\right)^{n+1}} c_{1} \sqrt{2}\left(\frac{r_{0}}{r}\right)^{n+1}-2 \sqrt{1-\left(\frac{r_{0}}{r}\right)^{n+1}} \alpha \sqrt{\left(-1+\sqrt{1-\left(\frac{r_{0}}{x}\right)^{n+1}}\right)^{2} r^{-2}}\left(\frac{r_{0}}{r}\right)^{n+1} n \\
& +2 \sqrt{1-\left(\frac{r_{0}}{r}\right)^{n+1}} \alpha \sqrt{\left(-1+\sqrt{1-\left(\frac{r_{0}}{r}\right)^{n+1}}\right)^{2} r^{-2}}\left(\frac{r_{0}}{r}\right)^{n+1}+3 \sqrt{1-\left(\frac{r_{0}}{r}\right)^{n+1}} c_{1} \sqrt{2} r\left(\frac{r_{0}}{r}\right)^{n+1} \\
& -\sqrt{1-\left(\frac{r_{0}}{r}\right)^{n+1}} c_{1} \sqrt{2}\left(\frac{r_{0}}{r}\right)^{n+1} n-8 \sqrt{1-\left(\frac{r_{0}}{r}\right)^{n+1}} \alpha \sqrt{\left(-1+\sqrt{1-\left(\frac{r_{0}}{r}\right)^{n+1}}\right)^{2} r^{-2}} \\
& -8 \sqrt{1-\left(\frac{r_{0}}{r}\right)^{n+1}} c_{1} \sqrt{2}+8 \alpha \sqrt{\left(-1+\sqrt{1-\left(\frac{r_{0}}{r}\right)^{n+1}}\right)^{2} r^{-2}} \\
& -8 \alpha \sqrt{\left(-1+\sqrt{1-\left(\frac{r_{0}}{r}\right)^{n+1}}\right)^{2} r^{-2}}\left(\frac{r_{0}}{r}\right)^{n+1}-2 \sqrt{1-\left(\frac{r_{0}}{r}\right)^{n+1}} c_{1} \sqrt{2} r+8 c_{1} \sqrt{2} \\
& -8 c_{1} \sqrt{2}\left(\frac{r_{0}}{r}\right)^{n+1}-2 \sqrt{\left(-1+\sqrt{1-\left(\frac{r_{0}}{r}\right)^{n+1}}\right)^{2} r^{-2}} \sqrt{1-\left(\frac{r_{0}}{r}\right)^{n+1}} c_{2} r^{2} \\
& \left.+2 c_{1} \sqrt{2} r-2 c_{1} \sqrt{2} r\left(\frac{r_{0}}{r}\right)^{n+1}\right) \text {. }
\end{aligned}
$$




$$
\begin{aligned}
& \rho+p_{t}=\frac{1}{32 \pi} \frac{1}{\sqrt{\left(-1+\sqrt{1-\left(\frac{r_{0}}{r}\right)^{n+1}}\right)^{2} r^{-2}}} \frac{1}{\sqrt{1-\left(\frac{r_{0}}{r}\right)^{n+1}}} r^{-2}\left(2 \sqrt{1-\left(\frac{r_{0}}{r}\right)^{n+1}} c_{1} \sqrt{2}\left(\frac{r_{0}}{r}\right)^{n+1} n r\right. \\
& +6 \sqrt{1-\left(\frac{r_{0}}{r}\right)^{n+1}} c_{1} \sqrt{2}\left(\frac{r_{0}}{r}\right)^{n+1}-4 \sqrt{1-\left(\frac{r_{0}}{r}\right)^{n+1}} \alpha \sqrt{\left(-1+\sqrt{1-\left(\frac{r_{0}}{r}\right)^{n+1}}\right)^{2} r^{-2}}\left(\frac{r_{0}}{r}\right)^{n+1} n \\
& +8 \sqrt{1-\left(\frac{r_{0}}{r}\right)^{n+1}} \alpha \sqrt{\left(-1+\sqrt{1-\left(\frac{r_{0}}{r}\right)^{n+1}}\right)^{2} r^{-2}}\left(\frac{r_{0}}{r}\right)^{n+1}+6 \sqrt{1-\left(\frac{r_{0}}{r}\right)^{n+1}} c_{1} \sqrt{2} r\left(\frac{r_{0}}{r}\right)^{n+1} \\
& -2 \sqrt{1-\left(\frac{r_{0}}{r}\right)^{n+1}} c_{1} \sqrt{2}\left(\frac{r_{0}}{r}\right)^{n+1} n-16 \sqrt{1-\left(\frac{r_{0}}{r}\right)^{n+1}} \alpha \sqrt{\left(-1+\sqrt{1-\left(\frac{r_{0}}{r}\right)^{n+1}}\right)^{2} r^{-2}} \\
& -12 \sqrt{1-\left(\frac{r_{0}}{r}\right)^{n+1}} c_{1} \sqrt{2}+16 \alpha \sqrt{\left(-1+\sqrt{\left.1-\left(\frac{r_{0}}{r}\right)^{n+1}\right)^{2} r^{-2}}\right.} \\
& -16 \alpha \sqrt{\left(-1+\sqrt{1-\left(\frac{r_{0}}{r}\right)^{n+1}}\right)^{2} r^{-2}}\left(\frac{r_{0}}{r}\right)^{n+1}-4 \sqrt{1-\left(\frac{r_{0}}{r}\right)^{n+1}} c_{1} \sqrt{2} r+12 c_{1} \sqrt{2} \\
& -12 c_{1} \sqrt{2}\left(\frac{r_{0}}{r}\right)^{n+1}-2 \sqrt{\left(-1+\sqrt{1-\left(\frac{r_{0}}{r}\right)^{n+1}}\right)^{2} r^{-2}} \sqrt{1-\left(\frac{r_{0}}{r}\right)^{n+1}} c_{2} r^{2}+4 c_{1} \sqrt{2} r
\end{aligned}
$$

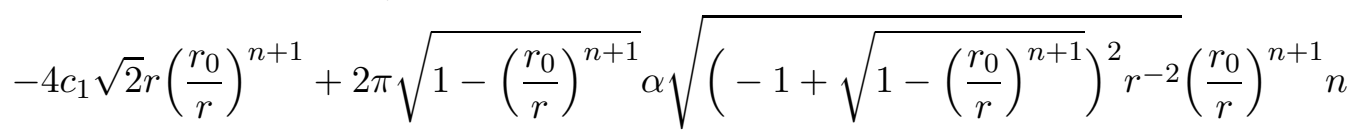

$$
\begin{aligned}
& +2 \pi \sqrt{1-\left(\frac{r_{0}}{r}\right)^{n+1}} \alpha \sqrt{\left(-1+\sqrt{1-\left(\frac{r_{0}}{r}\right)^{n+1}}\right)^{2} r^{-2}}\left(\frac{r_{0}}{r}\right)^{n+1} \\
& -2 \pi \sqrt{\left(-1+\sqrt{1-\left(\frac{r_{0}}{r}\right)^{n+1}}\right)^{2} r^{-2}} \sqrt{1-\left(\frac{r_{0}}{r}\right)^{n+1}} c_{2} r^{2}-4 \pi \sqrt{1-\left(\frac{r_{0}}{r}\right)^{n+1}} c_{1} \sqrt{2} \\
& +4 \pi c_{1} \sqrt{2}-4 \pi c_{1} \sqrt{2}\left(\frac{r_{0}}{r}\right)^{n+1}+\pi \sqrt{1-\left(\frac{r_{0}}{r}\right)^{n+1}} c_{1} \sqrt{2}\left(\frac{r_{0}}{r}\right)^{n+1} n \\
& \left.+3 \pi \sqrt{1-\left(\frac{r_{0}}{r}\right)^{n+1}} c_{1} \sqrt{2}\left(\frac{r_{0}}{r}\right)^{n+1}\right) .
\end{aligned}
$$

The qualitative behavior of the energy density; the radial pressure; and the tangential pressure is plotted in Fig.1, left panel. Note that the energy density is negative in entire spacetime. So the anisotropic case violates the WEC also NEC. But just for a small region which is denoted by $r<0.25$, the energy density is positive. So, just there is one possibility to have a micro or tiny wormholes but in any case as we observe in the right panel, the NEC violated. The NEC for $\left(p_{t}, p_{r}\right)$ must be check by positivity of the (2122). The violation of the NEC is for both cases of the radial and the tangential components of the pressure. Note that we adopted the parameters of our $f(T)$ to meet the observational data by cosmography technique. 

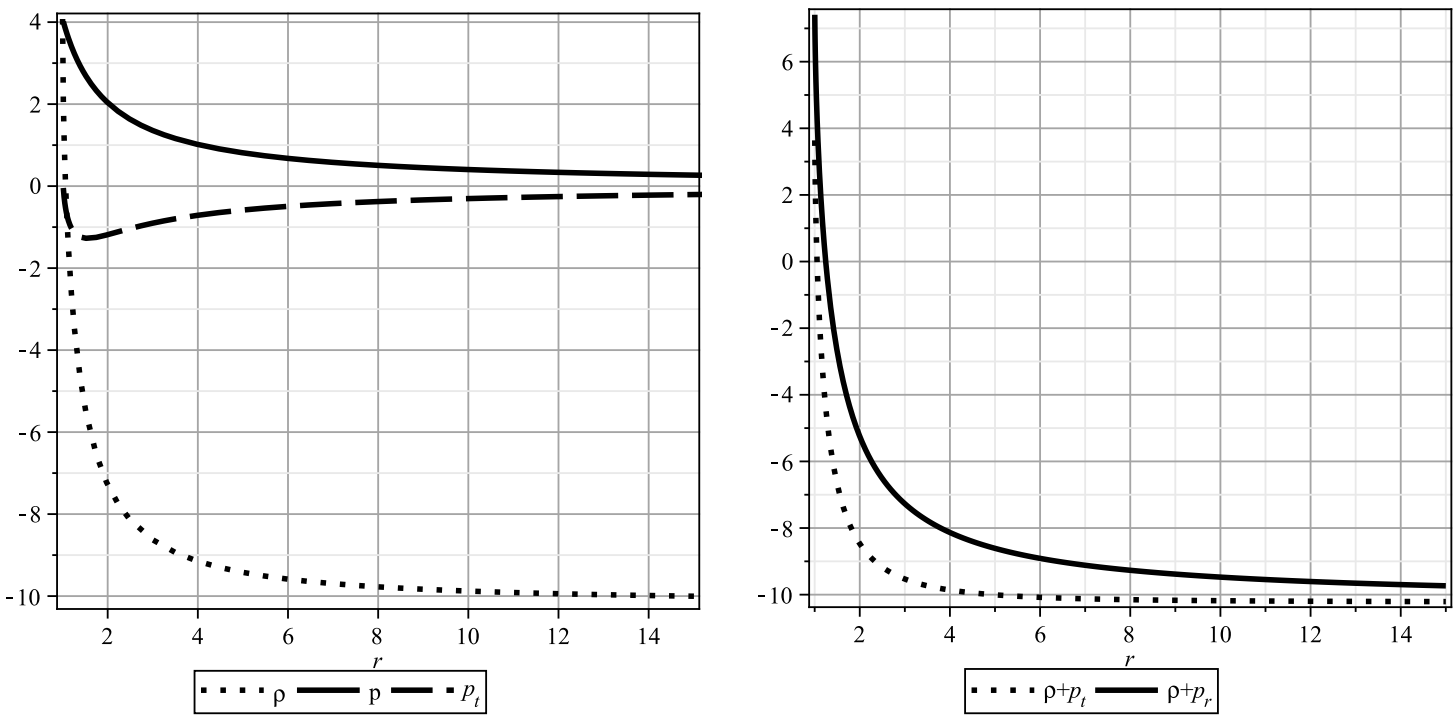

FIG. 1: (Left) The dotted curve shows the energy density; the solid the radial pressure; and the dashed curve the tangential pressure. We have defined the following quantities: $r_{0}=1, n=0.2, c_{1}=6^{1 / 2} / 2 H_{0}\left(\Omega_{m 0}-\right.$ 1), $c_{2}=\Omega_{m 0}, \alpha=0$. (Right) Variation of the $\rho+p_{r}$ solid, and $\rho+p_{t}$ dotted for the following quantities: $r_{0}=1, n=0.2, c_{1}=6^{1 / 2} / 2 H_{0}\left(\Omega_{m 0}-1\right), c_{2}=\Omega_{m 0}, \alpha=0$.

\section{SOLUTION FOR ISOTROPIC PRESSURE}

To obtain the isotropic solutions we assume that

$$
a(r)=c, \quad p_{r}(r)=p_{t}(r)=p(r) .
$$

Now from Eqs.(3)-(5), we obtain the following solutions

$$
b_{ \pm}(r)=2 \log \left[\frac{-1 \pm \sqrt{-r^{2} C+1}}{1-r^{2} C \mp \sqrt{-r^{2} C+1}}\right]
$$

where $C$ is a constant of integration. The asymptotic behavior of $\beta$ is

$$
\beta_{ \pm} \simeq C r^{3}+O\left(\frac{1}{r^{2}}\right)
$$

which diverges as $r \rightarrow \infty$. so the wormhole spacetime is not asymptotically flat in both cases of $b_{ \pm}$. Due to this reason, we cannot define the ADM mass of wormhole.

To find out the radius of wormhole's throat, Following [28], we consider the following line element

$$
d s^{2}=e^{2 \Phi(l)} d t^{2}-d l^{2}-r^{2}(l) d \Omega^{2}
$$


To obtain the radius of throat, we define the proper distance

$$
l(r)= \pm \int_{r_{0}}^{r} \frac{d r^{\prime}}{\sqrt{1-\frac{\beta_{ \pm}\left(r^{\prime}\right)}{r^{\prime}}}} .
$$

Solving the above integral, we obtain

$$
l(r)=\mp \frac{\arcsin (\sqrt{C} r)}{\sqrt{C}} .
$$

Now by taking inverse on both sides

$$
r=\mp \frac{1}{\sqrt{C}} \sin (l \sqrt{C}) .
$$

Now the radius of throat $r_{0}=\min \{r(l)\}$, is given by

$$
\left|r_{0}\right|=\frac{1}{\sqrt{C}}
$$

Finally $C=\frac{1}{r_{0}^{2}}$. By this solution we have the following functions for the energy density $\rho$, and the pressure $p(r)$ :

$$
\begin{aligned}
& 4 \pi \rho_{+}(r)=5 / 2\left(-3 / 5 r_{0}^{2}\left(r\left((-16-16 / 3 r) r_{0}^{6}+\left(28 r^{2}+\frac{20}{3} r^{3}\right) r_{0}^{4}+\left(-5 / 3 r^{5}-13 r^{4}\right) r_{0}^{2}+r^{6}\right) c_{1} \sqrt{2}\right.\right. \\
& \left.+\left(-8 / 3 c_{2} r^{2}-\frac{128}{3} \alpha\right) r_{0}{ }^{6}+10 / 3 r^{2}\left(\frac{136}{5} \alpha+c_{2} r^{2}\right) r_{0}{ }^{4}-5 / 6 r^{4}\left(c_{2} r^{2}+\frac{344}{5} \alpha\right) r_{0}{ }^{2}+\frac{29}{3} \alpha r^{6}\right) \\
& \times \sqrt{-\frac{r^{2}}{r_{0}^{2}}+1}+\left(-1 / 5 r c_{1}\left((16 r+48) r_{0}{ }^{4}+\left(-12 r^{3}-60 r^{2}\right) r_{0}{ }^{2}\right.\right. \\
& \left.+r^{4}(15+r)\right) r_{0}^{2} \sqrt{2}+\left(-8 / 5 c_{2} r^{2}-\frac{128}{5} \alpha\right) r_{0}^{6}+6 / 5 r^{2}\left(c_{2} r^{2}+\frac{104}{3} \alpha\right) r_{0}^{4} \\
& \left.\left.-1 / 10 r^{4}\left(c_{2} r^{2}+168 \alpha\right) r_{0}^{2}+\alpha r^{6}\right)\left(r+r_{0}\right)\left(-r_{0}+r\right)\right) \frac{1}{\sqrt{-\frac{r^{2}}{r_{0}^{2}}+1}} r_{0}^{-6} r^{-2}
\end{aligned}
$$

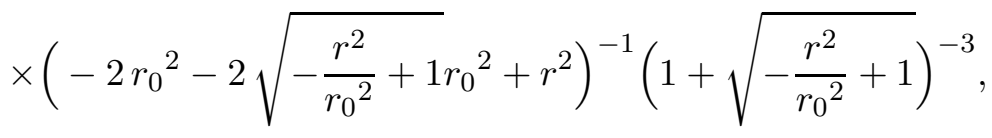

$$
\begin{aligned}
& 4 \pi p_{+}(r)=-1 / 2\left(\left(20 r^{2} r_{0}{ }^{4}-5 r^{4} r_{0}{ }^{2}-16 r_{0}{ }^{6}\right) \sqrt{-\frac{r^{2}}{r_{0}{ }^{2}}+1}-13 r^{4} r_{0}{ }^{2}+r^{6}+28 r^{2} r_{0}{ }^{4}-16 r_{0}{ }^{6}\right) \\
& \times\left(c_{1} \sqrt{2} r_{0}^{2}+r\left(1 / 2 c_{2} r_{0}^{2}+\alpha\right)\right) \frac{1}{\sqrt{-\frac{r^{2}}{r_{0}^{2}}+1}} r_{0}^{-6} r^{-1}\left(-2 r_{0}^{2}-2 \sqrt{-\frac{r^{2}}{r_{0}^{2}}+1} r_{0}^{2}+r^{2}\right)^{-1} \\
& \times\left(1+\sqrt{-\frac{r^{2}}{r_{0}{ }^{2}}+1}\right)^{-3} .
\end{aligned}
$$


So the expression for the NEC reads by the following expression:

$$
\begin{aligned}
& \rho_{+}+p_{+}=1 / 2\left(-3 / 4\left(c_{1} r\left(\left(-16 / 3 r-\frac{64}{3}\right) r_{0}{ }^{6}+\frac{20}{3} r^{2}\left(r+\frac{26}{5}\right) r_{0}^{4}+\left(-5 / 3 r^{5}-\frac{44}{3} r^{4}\right) r_{0}^{2}+r^{6}\right) \sqrt{2}\right.\right. \\
& \left.+\left(-\frac{128}{3} \alpha-16 / 3 c_{2} r^{2}\right) r_{0}{ }^{6}+\frac{20}{3} r^{2}\left(c_{2} r^{2}+\frac{64}{5} \alpha\right) r_{0}{ }^{4}-5 / 3 r^{4}\left(c_{2} r^{2}+\frac{152}{5} \alpha\right) r_{0}{ }^{2}+8 \alpha r^{6}\right) r_{0}{ }^{2} \\
& \times \sqrt{-\frac{r^{2}}{r_{0}^{2}}+1}+\left(r+r_{0}\right)\left(-r_{0}+r\right)\left(-1 / 4 c_{1} r\left((16 r+64) r_{0}{ }^{4}+\left(-72 r^{2}-12 r^{3}\right) r_{0}{ }^{2}\right.\right. \\
& \left.+r^{4}(16+r)\right) r_{0}{ }^{2} \sqrt{2}+\left(-32 \alpha-4 c_{2} r^{2}\right) r_{0}{ }^{6}+\left(48 \alpha r^{2}+3 c_{2} r^{4}\right) r_{0}{ }^{4}+\left(-18 r^{4} \alpha-1 / 4 r^{6} c_{2}\right) r_{0}{ }^{2}
\end{aligned}
$$

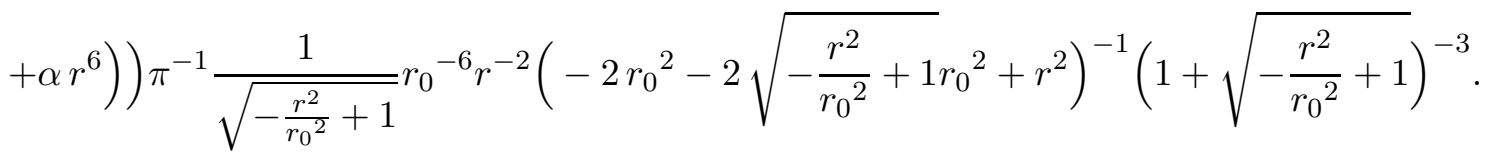

The qualitative behavior of the energy density and the pressure for positive branch is plotted in Fig.2, left panel. Note that the energy density remains positive. So the isotropic case satisfies the WEC. Further, as we observe in the right panel, also the NEC satisfies here by the EM components. The NEC is satisfied for both cases of the radial and the tangential components of the pressure. So there is at least one possibility to have a physically reasonable wormhole solution with isotropic pressures in our viable $f(T)$ model. Finally in the isotropic case, for negative branch, from figures 3 , both null and weak energy conditions are violated.

For minus branch:

$$
\begin{aligned}
& 4 \pi \rho_{-}(r)=5 / 2\left(3 / 5 r_{0}^{2}\left(c_{1} r\left((-16 / 3 r-16) r_{0}{ }^{6}+\left(\frac{20}{3} r^{3}+28 r^{2}\right) r_{0}^{4}+\left(-13 r^{4}-5 / 3 r^{5}\right) r_{0}^{2}+r^{6}\right) \sqrt{2}\right.\right. \\
& \left.+\left(-8 / 3 c_{2} r^{2}-\frac{128}{3} \alpha\right) r_{0}{ }^{6}+10 / 3 r^{2}\left(c_{2} r^{2}+\frac{136}{5} \alpha\right) r_{0}{ }^{4}-5 / 6\left(c_{2} r^{2}+\frac{344}{5} \alpha\right) r^{4} r_{0}{ }^{2}+\frac{29}{3} \alpha r^{6}\right) \\
& \times \sqrt{-\frac{r^{2}}{r_{0}{ }^{2}}+1}+\left(r+r_{0}\right)\left(-1 / 5 c_{1} r_{0}^{2} r\left((48+16 r) r_{0}^{4}+\left(-60 r^{2}-12 r^{3}\right) r_{0}^{2}\right.\right. \\
& \left.+r^{4}(15+r)\right) \sqrt{2}+\left(-\frac{128}{5} \alpha-8 / 5 c_{2} r^{2}\right) r_{0}{ }^{6}+6 / 5\left(c_{2} r^{2}+\frac{104}{3} \alpha\right) r^{2} r_{0}{ }^{4} \\
& \left.\left.-1 / 10 r^{4}\left(168 \alpha+c_{2} r^{2}\right) r_{0}^{2}+\alpha r^{6}\right)\left(-r_{0}+r\right)\right) \frac{1}{\sqrt{-\frac{r^{2}}{r_{0}^{2}}+1}} r_{0}^{-6} r^{-2}
\end{aligned}
$$

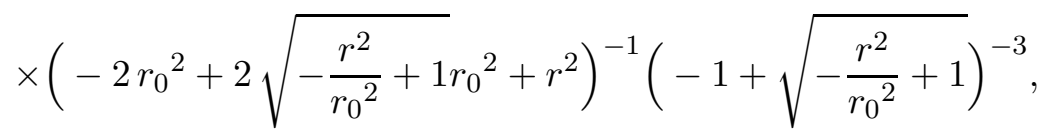

$$
\begin{aligned}
& 4 \pi p_{-}(r)=-1 / 2\left(c_{1} \sqrt{2} r_{0}{ }^{2}+r\left(1 / 2 c_{2} r_{0}^{2}+\alpha\right)\right)\left(\left(16 r_{0}{ }^{6}-20 r^{2} r_{0}{ }^{4}+5 r^{4} r_{0}^{2}\right) \sqrt{-\frac{r^{2}}{r_{0}^{2}}+1}-13 r^{4} r_{0}^{2}\right. \\
& \left.+r^{6}+28 r^{2} r_{0}^{4}-16 r_{0}^{6}\right) \frac{1}{\sqrt{-\frac{r^{2}}{r_{0}^{2}}+1}} r_{0}^{-6} r^{-1}\left(-2 r_{0}^{2}+2 \sqrt{-\frac{r^{2}}{r_{0}^{2}}+1} r_{0}^{2}+r^{2}\right)^{-1} \\
& \times\left(-1+\sqrt{-\frac{r^{2}}{r_{0}^{2}}+1}\right)^{-3}
\end{aligned}
$$


and the NEC reads

$$
\begin{aligned}
\rho_{-}+p_{-}= & 1 / 2\left(3 / 4 r_{0}^{2}\left(c_{1} r\left(\left(-\frac{64}{3}-16 / 3 r\right) r_{0}{ }^{6}+\frac{20}{3}\left(r+\frac{26}{5}\right) r^{2} r_{0}^{4}+\left(-\frac{44}{3} r^{4}-5 / 3 r^{5}\right) r_{0}^{2}+r^{6}\right) \sqrt{2}\right.\right. \\
& \left.+\left(-\frac{128}{3} \alpha-16 / 3 c_{2} r^{2}\right) r_{0}^{6}+\frac{20}{3} r^{2}\left(\frac{64}{5} \alpha+c_{2} r^{2}\right) r_{0}{ }^{4}-5 / 3\left(c_{2} r^{2}+\frac{152}{5} \alpha\right) r^{4} r_{0}{ }^{2}+8 \alpha r^{6}\right) \\
& \times \sqrt{-\frac{r^{2}}{r_{0}^{2}}+1+\left(r+r_{0}\right)\left(-1 / 4 r_{0}^{2}\left((16 r+64) r_{0}^{4}+\left(-12 r^{3}-72 r^{2}\right) r_{0}^{2}\right.\right.} \\
& \left.+r^{4}(16+r)\right) c_{1} r \sqrt{2}+\left(-4 c_{2} r^{2}-32 \alpha\right) r_{0}^{6}+\left(3 c_{2} r^{4}+48 r^{2} \alpha\right) r_{0}^{4} \\
& \left.\left.+\left(-1 / 4 r^{6} c_{2}-18 r^{4} \alpha\right) r_{0}^{2}+\alpha r^{6}\right)\left(-r_{0}+r\right)\right) \pi^{-1} \frac{1}{\sqrt{-\frac{r^{2}}{r_{0}^{2}}+1}} r_{0}^{-6} r^{-2} \\
& \left(-2 r_{0}^{2}+2 \sqrt{\left.-\frac{r^{2}}{r_{0}^{2}}+1 r_{0}^{2}+r^{2}\right)^{-1}\left(-1+\sqrt{-\frac{r^{2}}{r_{0}^{2}}+1}\right)^{-3}} .\right.
\end{aligned}
$$
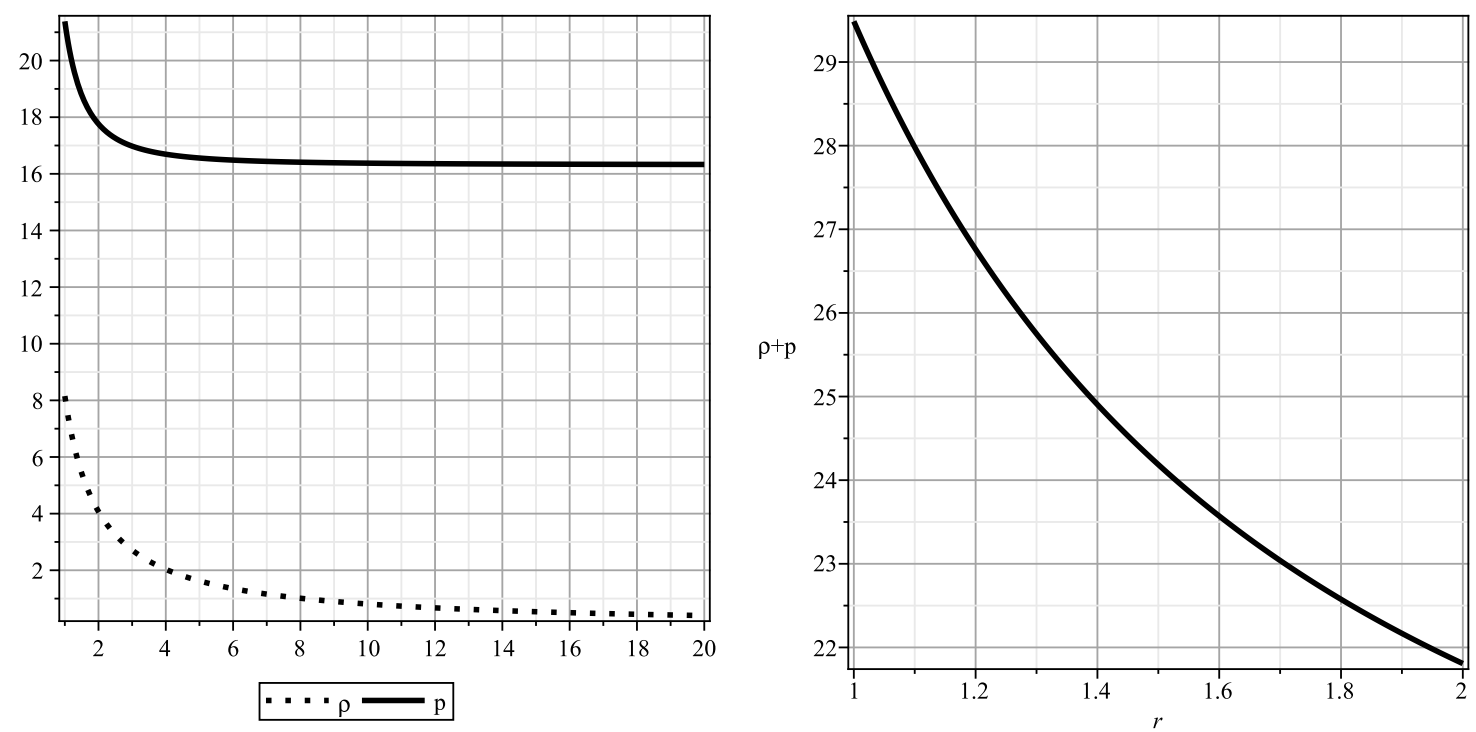

FIG. 2: (Left)Positive Branch: The dotted curve for the energy density; the solid as radial pressure. We have defined the following quantities: $r_{0}=1, n=0.2, c_{1}=6^{\frac{1}{2}} / 2 H_{0}\left(\Omega_{m 0}-1\right), c_{2}=\Omega_{m 0}, \alpha=0$. (Right) Variation of the $\rho+p_{r}$ for the following quantities: $r_{0}=1, n=0.2, c_{1}=6^{\frac{1}{2}} / 2 H_{0}\left(\Omega_{m 0}-1\right), c_{2}=\Omega_{m 0}, \alpha=0$.

\section{SOLUTION WITH BAROTROPIC EQUATION OF STATE}

In this case the exact solution exists only for $\alpha=0$. We assume

$$
a(r)=c, \quad p_{r}(r)=k \rho(r)
$$



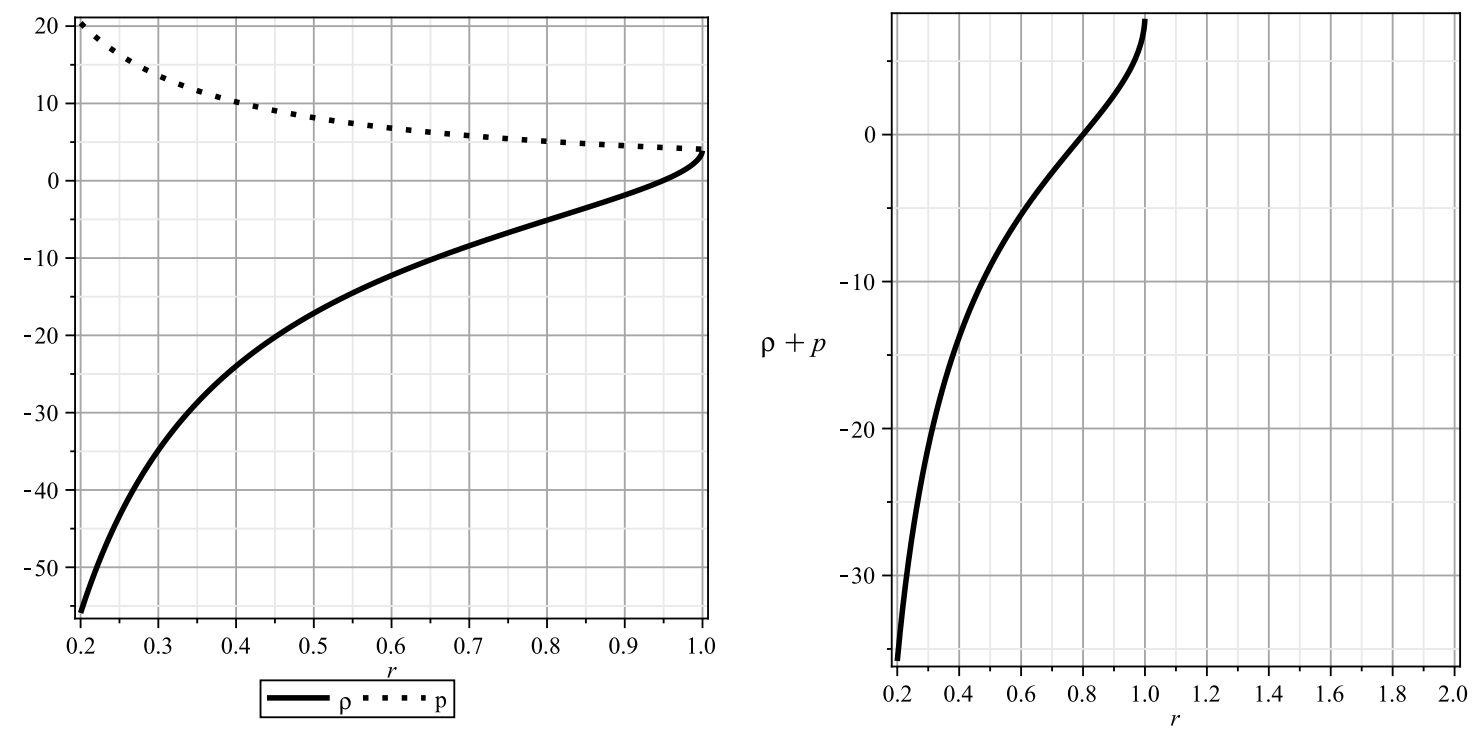

FIG. 3: (Left) Minus branch:The solid curve is for the energy density; the dotted indicates the radial pressure. We have defined the following quantities: $r_{0}=1, n=0.2, c_{1}=6^{\frac{1}{2}} / 2 H_{0}\left(\Omega_{m 0}-1\right), c_{2}=\Omega_{m 0}, \alpha=0$. (Right) Variation of the $\rho+p_{r}$ for the following quantities: $r_{0}=1, n=0.2, c_{1}=6^{\frac{1}{2}} / 2 H_{0}\left(\Omega_{m 0}-1\right), c_{2}=$ $\Omega_{m 0}, \alpha=0$.

where $k$ is an arbitrary but finite constant. Using (33) in the equations of motion, we obtain

$$
\begin{aligned}
b(r)= & 2 \log \left(\frac{r^{2}}{r^{2}+C\left(1-3 r+3 r^{2}-r^{3}\right)}\right), \\
4 \pi \rho(r)= & -\frac{c_{2} r^{6}+2 c_{1} \sqrt{2} r^{5}}{4 r^{6}}, \\
4 \pi p_{r}(r)= & -k \frac{c_{2} r^{6}+2 c_{1} \sqrt{2} r^{5}}{4 r^{6}}, \\
4 \pi p_{t}(r)= & \frac{1}{4 r^{6}(-1+r)}\left(-c_{2} r^{7}+c_{2} r^{6}+3 C \sqrt{2} c_{1} r^{6}-c_{1} \sqrt{2} r^{6}-9 C \sqrt{2} c_{1} r^{5}\right. \\
& \left.-2 c_{1} \sqrt{2} r^{5}+9 C \sqrt{2} c_{1} r^{4}-3 C \sqrt{2} c_{1} r^{3}\right) .
\end{aligned}
$$

So, the expression for the transverse null energy condition $\rho+p_{t}>0$ reads

$$
\begin{aligned}
\rho+p_{t}= & \frac{1}{16 \pi r^{3}(1-r)}\left(2 c_{2} r^{4}+\left(-2 c_{2}+3 c_{1} \sqrt{2}-3 C \sqrt{2} c_{1}\right) r^{3}+9 C \sqrt{2} c_{1} r^{2}\right. \\
& \left.-9 C \sqrt{2} c_{1} r+3 C \sqrt{2} c_{1}\right) .
\end{aligned}
$$

Again, the qualitative behavior of the energy density and the pressure is plotted in Fig.4, left panel, in $\log$ scale. Note that the energy density is positive. So the isotropic case satisfies the WEC. Further, as we observe in the right panel, the NEC is satisfied by the EM tensor components. The non violation of the NEC is for the tangential component of the pressure. So there exists at least one possibility to have a physically acceptable wormhole solution with barotropic radial fluid in 
our viable $f(T)$ model. In fact, in the barotropic case, obviously the NEC is satisfied entirely the spacetime. In figure-5, we plot the ratio of pressure and energy density and show that these remain positive for asymptotic values of $r$.
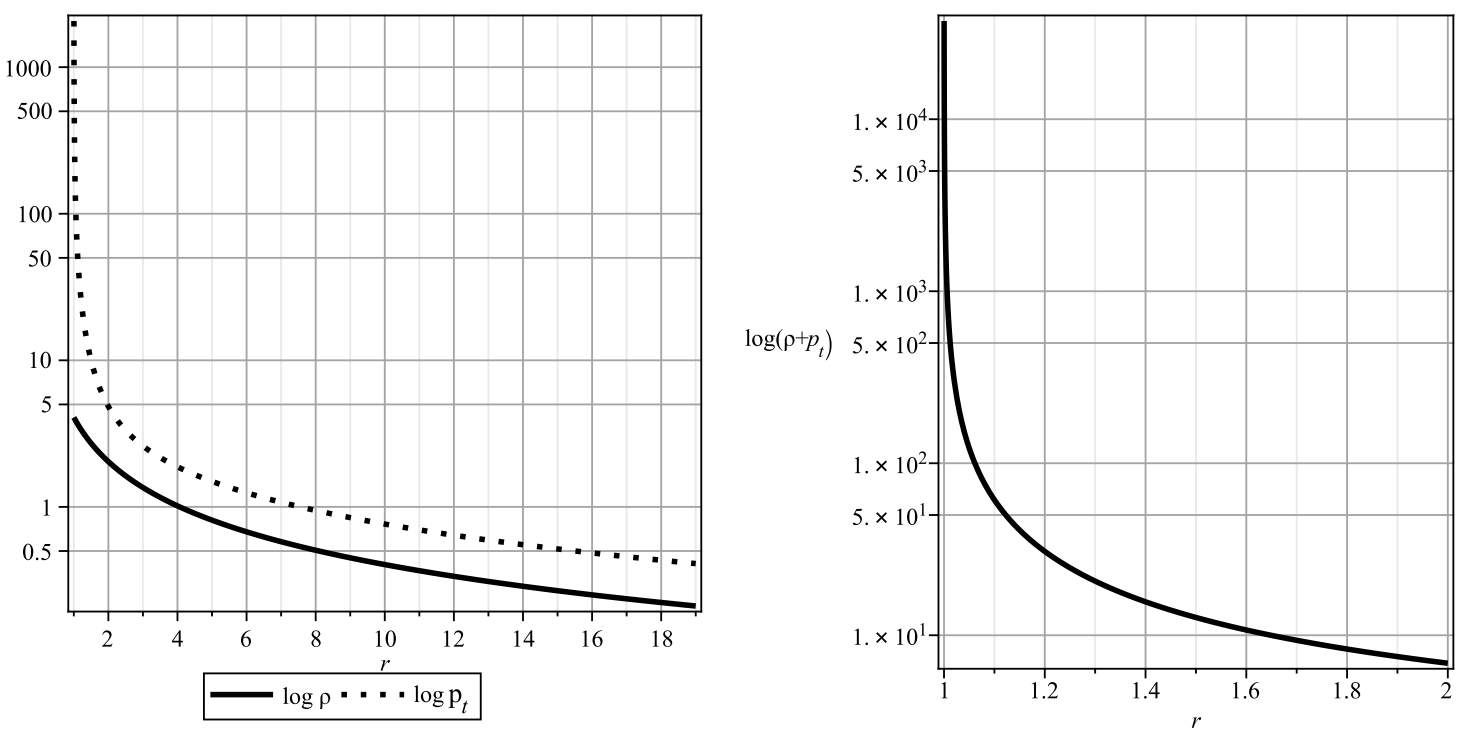

FIG. 4: (Left) We have defined the following quantities: $r_{0}=1, n=0.2, c_{1}=6^{\frac{1}{2}} / 2 H_{0}\left(\Omega_{m 0}-1\right), c_{2}=$ $\Omega_{m 0}, \alpha=0$. (Right) Variation of the $\log \left(\rho+p_{t}\right)$ for the following quantities: $r_{0}=1, n=0.2, c_{1}=$ $6^{\frac{1}{2}} / 2 H_{0}\left(\Omega_{m 0}-1\right), c_{2}=\Omega_{m 0}, \alpha=0$.

\section{CONCLUSION}

We have considered Morris -Thorne wormholes, i.e., static and spherically symmetric traversable wormholes, in the Weitzenbock spacetime with torsion. Basically, we discussed the possible wormhole solutions in a viable $f(T)$ model with form $f(T)=2 c_{1} \sqrt{-T}+\alpha T+c_{2}$. This model was picked up for its simplicity in the numerical computation and astrophysical viability. We investigated three kinds of the fluids including isotropic, anisotropic and finally the barotropic fluids. We presented specific solutions with various choices of the shape function. In all cases, we obtained the exact solutions which described the wormhole geometries. By checking the behavior of the weak and null energy conditions for each case, we observed their violation for anisotropic case while their satisfaction for isotropic and barotropic cases. So we can have both isotropic and barotropic wormhole solutions in this viable torsion based model of the gravity.

In anisotropic case, our $f(T)$ model mimics the phantom energy since both energy conditions NEC and WEC are violated $r>r_{0}$. In isotropic case, we have two special cases for shape function. 

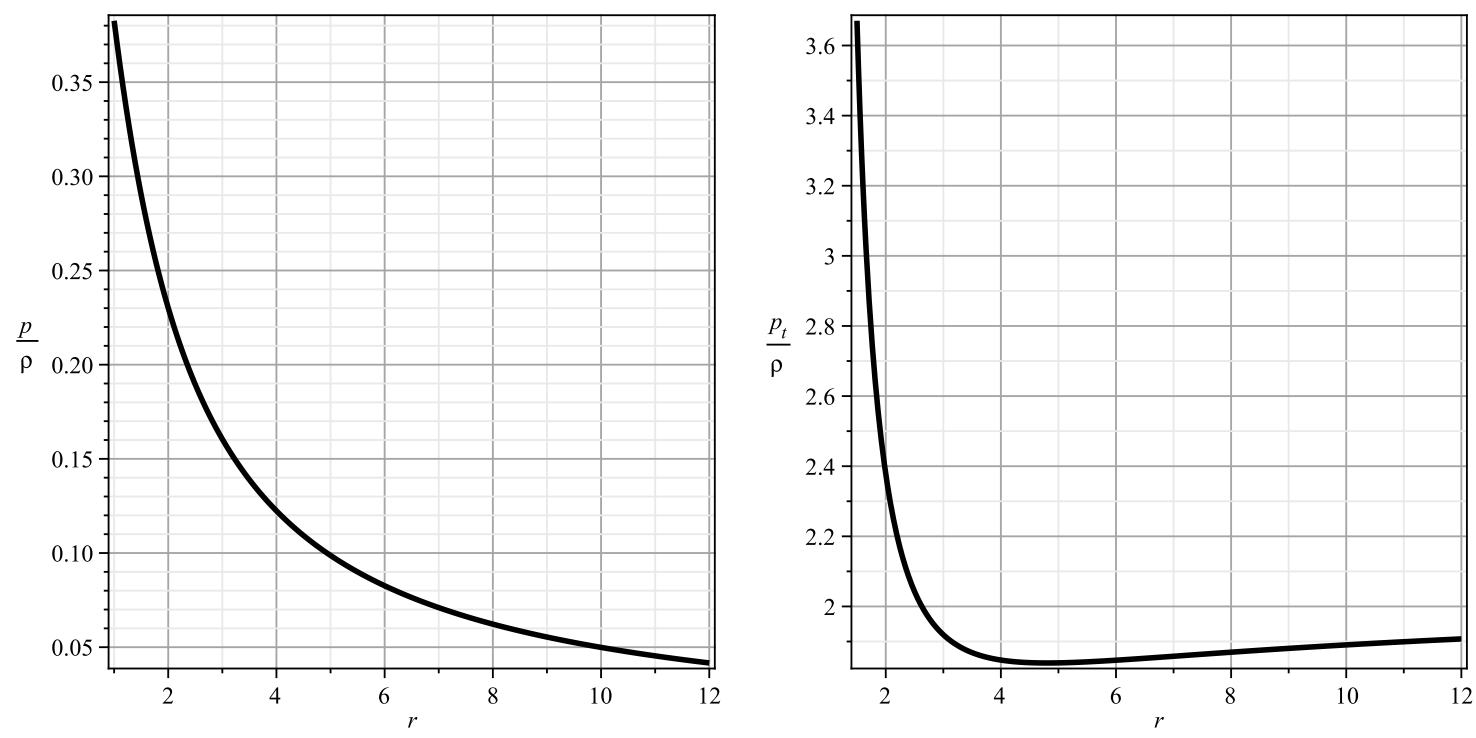

FIG. 5: (Left) The curve depicts the EoS parameter $w=\frac{p}{\rho}$ for isotropic case. We have defined the following quantities: $r_{0}=1, n=0.2, c_{1}=6^{1 / 2} / 2 H_{0}\left(\Omega_{m 0}-1\right), c_{2}=\Omega_{m 0}, \alpha=0$. (Right) Variation of the EoS parameter $w=\frac{p_{t}}{\rho}$ for barotropic case for the following quantities: $r_{0}=1, n=0.2, c_{1}=6^{1 / 2} / 2 H_{0}\left(\Omega_{m 0}-\right.$ 1), $c_{2}=\Omega_{m 0}, \alpha=0$.

For positive branch, both energy conditions are satisfied while they are violated in the negative branch case. Also the obtained wormhole solution is not asymptotically flat. For barotropic case, again we have non-asymptotically flat solution and energy conditions are satisfied for transverse NEC and WEC. Moreover, we discussed the behavior of the EoS parameter $w=p / \rho$ for isotropic and barotropic cases. Our numerical simulation shows that for isotropic case, $w$ remains positive. While for barotropic case, the same behavior happens.

[1] V. C. de Andrade, J. G. Pereira, Phys. Rev D 56, 4689 (1997); F. W. Hehl, J. D. McCrea, E. W. Mielke, Y. Neeman, Phys. Rep. 258, 1 (1995).

[2] F.S.N. Lobo, Classical and Quantum Gravity Research, 1-78, (2008), Nova Sci. Pub. ISBN 978-1-60456366-5; arXiv:0710.4474 [gr-qc].

[3] M.S. Morris and K.S. Thorne, Am. J. Phys. 56 (1988) 395; M.S. Morris et al, Phys. Rev. Lett. 61 (1988) 1446.

[4] M. Jamil, Int. J. Theor. Phys.49, 1549 (2010); M. Jamil, Nuovo Cim. B 123, 599 (2008); M. Jamil, M.U. Farooq, M.A. Rashid, M.A. Rashid, Eur. Phys. J. C 59, 907 (2009); M. Jamil, P. K.F. Kuhfittig, F. Rahaman, S. A Rakib, Eur. Phys. J. C 67 (2010) 513-520; M. Jamil, M. U. Farooq, Int. J. Theor. Phys. 49 (2010) 835-841; M. U. Farooq, M. Akbar, M. Jamil, AIP Conf. Proc. 1295 (2010) 176-190 
[5] P.K.F. Kuhfittig, Am. J. Phys. 67 (1999) 125.

[6] L.A. Anchordoqui et al, Phys. Rev. D 57 (1998) 829; B. Bhawal and S. Kar, Phys. Rev. D 46 (1992) 2464; D. Hochberg, Phys. Lett. B 251 (1990) 349; L. A. Anchordoqui, gr-qc/9612056; F. Rahaman et al, Nuovo Cim. B 121 (2006) 303; R.A. Konoplya and C. Molina, Phys. Rev D 71 (2005) 124009; S. Chakraborty and T. Bandyopadhyay, arXiv:0707.1181 [gr-qc]; K.A. Bronnikov and Sung-Won Kim, Phys. Rev. D 67 (2003) 064027.

[7] R.R. Caldwell, Phys. Lett. B 545 (2002) 23.

[8] S. Nojiri, S. D. Odintsov, Phys. Rept. 505, 59 (2011); S. Capozziello, M. De Laurentis, arXiv:1108.6266; T. Clifton, P. G. Ferreira, A. Padilla, C. Skordis, arXiv:1106.2476.

[9] C. Rovelli, liv. Rev. Rel.1, 1 (1998); A. Ashtekar, J. Lewandowski, Class. Quantum. Grav. 21, R53 (2004); M. Jamil, D. Momeni, M.A. Rashid, Eur. Phys. J. C 71, 1711 (2011); M. Jamil, U. Debnath, Astrophys. Space Sci. 333, 3 (2011); D. Dwivedee, B. Nayak, M. Jamil, L.P. Singh, arXiv:1110.6350.

[10] R. Maartens , Phys. Rev. D 62, 084023 (2000); R. Maartens, Living Rev. Relativity 7, 7 (2004).

[11] R. Kerner, Gen. Relativ. Gravit. 14, 453 (1982); G. Allemandi, A. Borowiec, M. Francaviglia, Phys. Rev. D 70, 103503 (2004); S.M. Carroll, A.D. Felice, V.Duvvuri, D.A. Easson, M. Trodden, M.S. Turner, Phys. Rev. D 71, 063513 (2005); T.P. Sotiriou, V. Faraoni, gr-qc/0805.1726; M. Jamil, D. Momeni, M. Raza, R. Myrzakulov, Eur. Phys. J. C 72:1999 (2012) arXiv:1107.5807; M. Jamil, F. M. Mahomed, D. Momeni, Phys.Lett.B702:315-319,2011 arXiv:1105.2610 M. R. Setare, D. Momeni , Int J Theor Phys (2012) 51:198-205 arXiv:1009.0918; M. R. Setare, D. Momeni, Mod.Phys.Lett.A26:151-159,2011 arXiv:1002.0185, M. R. Setare, D. Momeni, Int.J.Theor.Phys.50:106-113,2011, arXiv:1001.3767; M. R. Setare, D. Momeni, Int.J.Mod.Phys.D19:2079-2094,2010 , arXiv:0911.1877; D.Momeni , Int.J.Theor.Phys.50:1493-1514,2011 arXiv:0910.0594 ; A. Azadi, D. Momeni, M. Nouri-Zonoz, Phys.Lett.B670:210-214,2008 arXiv:0810.4673 D. Momeni, H. Gholizade, Int.J.Mod.Phys.D18:17191729,2009 ; arXiv:0903.0067; M. Jamil, D. Momeni, R. Myrzakulov, Chin. Phys. Lett. Vol. 29, No. 10 (2012) 109801, arXiv:1209.2916.

[12] M. Jamil, D. Momeni, R. Myrzakulov, European Physical Journal C 72:2137(2012); M. Jamil, D. Momeni, R. Myrzakulov,European Physical Journal C 72:2122(2012) arXiv:1209.1298, M. Jamil, D. Momeni, R. Myrzakulov, P. Pudra, J. Phys. Soc. Jpn. 81 (2012) 114004; M. Jamil,K. Yesmakhanova, D. Momeni, R. Myrzakulov,Cent. Eur. J. Phys. 10(5)(2012)1065-1071; M. J. S. Houndjo, D. Momeni, R. Myrzakulov,International Journal of Modern Physics D, DOI: 10.1142/S0218271812500939, arXiv:1206.3938, M. Jamil, D. Momeni, R. Myrzakulov, P. Rudra, J. Phys. Soc. Japan 81, 114004 (2012).

[13] M. Li, R-X Miao, Y-G Miao, JHEP 1107, 108 (2011).

[14] R. Miao, M. Li, Y. Miao, JCAP 11, 033 (2011).

[15] B. Li, T. P. Sotiriou, J. D. Barrow, Phys. Rev. D 83, 064035 (2011).

[16] K. Hayashi, T. Shirafuji, Phys. Rev. D 19, 3524 (1979); K. Hayashi, T. Shirafuji, Phys. Rev. D 24, 3312 (1981). 
[17] F. Hehl, P. von der Heyde, G. Kerlick, Rev. Mod. Phys. 48, 393 (1976).

[18] R.Ferraro, F.Fiorini, Phys. Rev. D 75, 084031 (2007); R. Ferraro, F. Fiorini, Phys. Rev. D 78, 124019 (2008); E. V.Linder, Phys. Rev. D 81127301 (2010).

[19] C. G. Boehmer, T. Harko, F. S. N. Lobo, Phys. Rev. D 85, 044033 (2012); J. B. Dent, S. Dutta, E. N. Saridakis, JCAP 1101 (2011) 009. arXiv:1010.2215 ; S. -H. Chen, J. B. Dent, S. Dutta, E. N. Saridakis, Phys. Rev. D83 (2011) 023508. arXiv:1008.1250 ; R. Zheng, Q. -G. Huang, JCAP 1103 (2011) 002 arXiv:1010.3512 ; T. P. Sotiriou, B. Li, J. D. Barrow, Phys. Rev. D83 (2011) 104030. arXiv:1012.4039 ; B. Li, T. P. Sotiriou, J. D. Barrow, Phys. Rev. D83 (2011) 104017, arXiv:1103.2786 ; G. R. Bengochea, Phys. Lett. B695 (2011) 405-411, arXiv:1008.3188; P. Wu, H. W. Yu, Phys. Lett. B693 (2010) 415-420, arXiv:1006.0674; R. -J. Yang, Europhys. Lett. 93 (2011) 60001, arXiv:1010.1376; Y. -F. Cai, S. - H. Chen, J. B. Dent, S. Dutta, E. N. Saridakis, arXiv:1104.4349; H. Wei, X. -P. Ma, H. -Y. Qi, arXiv:1106.0102; Y. Zhang, H. Li, Y. Gong, Z. -H. Zhu, JCAP 1107 (2011) 015, arXiv:1103.0719

[20] J. Wang et al., Phys. Lett. B 689, 133 (2010).

[21] S. W. Hawking, G.F.R. Ellis, The Large Scale Structure of Space-Time, (Cambridge University Press, 1973)

[22] N. M. Garci'a, T. Harko, F. S. N. Lobo, J. P. Mimoso, J. Phys. Conf. Ser. 314, 012060 (2011).

[23] Y. Gong, A. Wang, Phys. Lett. B 652, 63 (2007).

[24] M. S. Morris and K. S. Thorne, Am. J. Phys. 56, 395 (1988); M. S. Morris, K. S. Thorne, Ulvi. Yurtsever, Phys. Rev. Lett. 61, 1446 (1988).

[25] S. H. Hendi, D. Momeni ,Eur. Phys. J. C 71 (2011) 1823 arXiv:1201.0061.

[26] S.W. Hawking and G.F.R. Ellis, "The Large Scale Structure of Spacetime"', (Cambridge University Press, Cambridge 1973).

[27] M. Jamil, D. Momeni, R. Myrzakulov, Eur. Phys. J. C 72, 1959 (2012) arXiv:1202.4926.

[28] M. Visser, Lorentzian Wormholes: From Einstein to Hawking, American Institute of Physics; 1 edition (August 9, 1996) 